\title{
Editorial: Adapting assessment and feedback strategies
}

\author{
Gita Sedghi \\ University of Liverpool
}

Assessment should be aligned with courses' aims and module learning outcomes and used to improve student learning; therefore, constructive and timely feedback is essential for effective learning. During the pandemic, the transition to online learning and teaching called upon practitioners to investigate new ways to support and connect with their students. Since student engagement became a dilemma, assessment played an instrumental part in measuring student learning and participation to inform teachers and students of classroom learning. Consequently, feedback became even more crucial to mitigate learning gaps.

This section highlights the challenges learning developers and educators encountered to facilitate a supportive online learning community to allow students to benefit from each other's insights and create accessible and flexible assessments to help diverse cohorts. It showcases various assessment and feedback methods implemented to foster student engagement. However, the increased working demands of transitioning online meant that any measures taken did not add extra tasks on top of the already heavier workload for teaching staff. The authors explain the challenges of using eTools to find suitable platforms for their activities while demonstrating the need to be mindful that eTools dictate neither the pedagogy nor the development of assessment. Since the chosen pedagogical approach directly influences learning, the educators ensured that online and active assessment pedagogy was not compromised.

The reflections in this section present a range of initiatives that authors applied in response to the challenges brought by Covid-19. They demonstrate how crucial it is to ensure that assessments are authentic, with student perceptions of authenticity being vital and contributing significantly to the completion of learning outcomes. Pre-recorded videos to prepare for exams, collaborative student support network, video feedback, evaluation skills developed through the group evaluation activity, Vlog, minimising academic 
dishonesty issues and online peer-feedback are examples of activities to foster more meaningful assessment and feedback. The authors exhibit several ideas for the creation of authentic assessments to support online and active learning, increase student engagement and provide learners with a platform to demonstrate their skills.

There are a number of recommendations for educators in this section, including providing opportunities for students to engage in assessment design and to test their own ability to reach module learning outcomes by replicating summative assessment; giving timely feedback to improve future performance and student learning to enable a supportive environment for students to engage with the course content. Using different feedback modalities is feasible across the higher education sector, but care must be taken to support educators in developing competencies, providing clear instructions for access, listening to recipients' feedback and making adaptations so that feedback is meaningful and encouraging, motivating learners to improve their work. Educators must ensure that pedagogy takes priority; the tools should allow staff to support learning and develop resources that remain authentic to the agreed approach. 